\title{
Band Moiré Images
}

\author{
Roger David Hersch, Sylvain Chosson
}

\author{
Ecole Polytechnique Fédérale de Lausanne (EPFL), Switzerland
}

\begin{abstract}
We propose a new powerful way of synthesizing moiré images that enables the creation of dynamically moving messages incorporating text, symbols, and color elements. Moiré images appear when superposing a base layer made of replicated base bands and a revealing layer made of a line grating comprising thin transparent lines. Each replicated base band contains the same image, e.g. text or color motifs. Since the base bands and the revealing line grating have similar periods, the revealed moiré image is the image located within each base band, enlarged along one dimension. By considering the formation of the moiré image as a line sampling process, we derive the linear transformation between the base layer and the moiré image. We obtain the geometric layout of the resulting moiré image, i.e. its orientation, size and displacement direction when moving the revealing layer on top of the base layer. Interesting moiré images can be synthesized by applying geometric transformations to both the base and the revealing layers. We propose a mathematical model describing the geometric transformation that a moiré image undergoes, when its base layer and its revealing layer are subject to different freely chosen non-linear geometric transformations. By knowing in advance the layout of a moiré image as a function of the layouts of the base layer and of the revealing layer, we are able to create moiré components running up and down at different speeds and orientations upon translation of the revealing layer. We also derive layer transformations which yield periodic moiré images despite the fact that both the base and the revealing layers are curved. By offering a new means of artistic expression, band moiré images can be used to create new designs and to synthesize visually appealing applications.
\end{abstract}

Keywords: Optical art, moiré effect, 2D moirés images, band moirés, layer transformations, moiré transformation.

\section{Introduction}

Intriguing geometric patterns appear at the intersection of repetitive structures such as superposed combs, bridge side railings, superposed layers of fabric, curtains, etc.... These patterns result from interferences between repetitive layers and are known as moiré effects. The superposition of repetitive structures formed of alternating black and white lines (line gratings) yields alternating dark and highlight moiré lines known as moiré fringes (Fig. 1a). Line gratings are formed of replicated lines, each line having a specific transversal periodic 1D intensity profile (e.g. a cosinusoidal profile or in the case of bilevel lines a rectangular profile).

The geometry of moiré fringes has been widely studied (see section 2). Moiré fringes may be used for the analysis of deformations

RD.Hersch@epfl.ch, Sylvain.Chosson@epfl.ch

Proc. SIGGRAPH 2004,

ACM Trans. on Graphics,

Vol. 23, No.3, 239-248

of materials [Durelli and Parks, 1970], [Post 1994] as well as for the acquisition of 3D object shapes [Takasaki 1970].

(a)

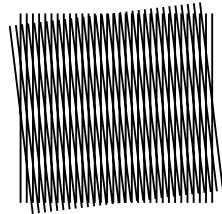

(b)
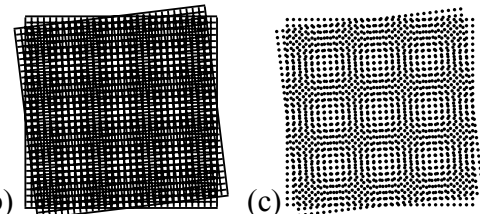

Fig. 1. Moiré fringes induced (a) by the superposition of two line gratings (b) by the superposition of two square grids and (c) by the superposition of two dot screens

In color reproduction, the ink layers form dot screens having specific periods and orientations. Moiré patterns may arise when superposing the cyan, magenta, yellow, and black dot screens [Yule 1967, Chapter 13]. A dot screen can be assimilated to a pair of superposed line gratings. Therefore, the geometric properties of moirés (orientation, period) induced by superpositions of dot screens (Fig. 1c) are the same as the geometric properties of the moiré fringes (Fig. 1a) induced by corresponding superposed line gratings [Amidror 2000].

A dot screen need not necessarily be composed of circular, elliptic or diamond shapes. Dot screens may be composed of replications of small squares (or parallelograms) incorporating a given pattern such as a micro-letter or a 2D micro-image. Mikami [1975] discovered that an array made of replicated tiny patterns sampled with an array of microlenses yields a moiré image comprising the enlarged and rotated array of tiny patterns (Fig. 2). Hutley, Hunt, Stevens and Savander [1994] computed how much the original array gets magnified and rotated as a function of the relative periods and orientations of the two layers.

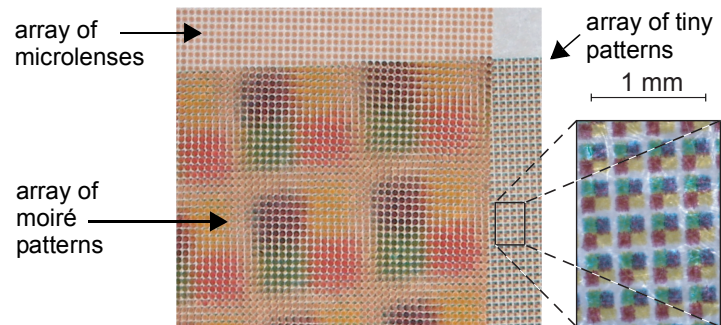

Fig. 2. Moiré generated by superposing an array of color patterns and an array of microlenses

Amidror [1994] observed that the dot intensity profiles of the replicated dots within the superposed dot screen layers determine the moiré intensity profile. By analyzing this phenomenon in Fourier space, he concluded that the moiré intensity profile is the cyclic convolution [Bracewell 1986, p. 362-363] of the normalized dot intensity profiles.

However, the "moiré magnifier" [Hutley et. al. 1994] made of a dot screen revealed by an array of tiny dots [Amidror 2002] or of microlenses offers only limited capabilities. Only a few characters or symbols can be incorporated within the location of a single dot, i.e. the revealed moiré consists of a very simple repetitive pattern 
(Fig. 2). In addition, with a revealing layer made of tiny holes, very little light is able to pass through these holes, allowing the moiré to be visible only in transparent mode in front of a strong light source.

We observed that when superposing a color line grating whose 1D intensity profile comprises a succession of different colors and a revealing line grating printed on a transparent film, the resulting moire fringes are formed by enlarged and rotated instances of the original succession of colors (Fig. 3a).

Since much more light passes through a line grating of a given period and relative aperture than through a dot screen of the same period and of the same relative aperture as dot diameter, 1D moiré images induced by line gratings have a much higher dynamic range than 2D moirés images obtained by superposing a dot screen and an array of tiny holes. We therefore propose to keep the revealing line grating as revealing layer and to introduce as base layer a base band grating made of replicated bands comprising freely chosen flat patterns or flat images (Fig. 3b). A base band grating differs from a line grating by having instead of a $1 \mathrm{D}$ intensity profile a $2 \mathrm{D}$ intensity profile, i.e. an intensity profile which varies according to the current position both in the transversal and in the longitudinal line directions. A base band becomes a full 2D image of its own, which can be revealed by superposing on the corresponding base band grating a revealing layer made of thin transparent lines.

And indeed, as will be described in section 3, periodic bands comprising vertically compressed flat patterns revealed by a line grating yield a moiré layer with periodic moiré image bands comprising vertically enlarged and transformed instances of the original flat patterns (Fig. 3b).

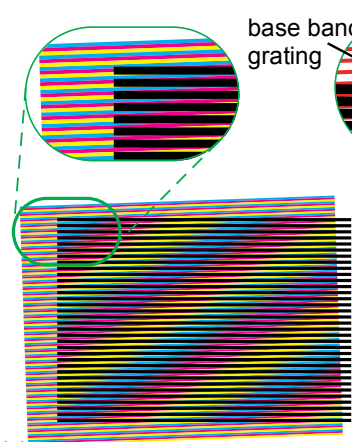

(a)

Fig. 3. (a) Color moiré fringes and (b) band moiré image generated by superposing a base band grating made of a replicated, vertically compressed text and a revealing line grating.

A further advantage of using base bands lies in the fact that different design elements (text, graphics, grayscale or color motives) may be freely laid out along the length of the base band. The longer the base band, the more elements it can incorporate.

In order to fully exploit the fact that moiré images may enlarge the flat, possibly colored patterns embedded into the base layer's base bands, a first challenge was to develop a model describing the geometric transformation between the base band space and the moiré image space. This geometric transformation specifies the mapping of each point of the base band space into the moire image space, as a function of the geometric layouts of both the base and the revealing layers. We may also deduce from that geometric transformation the orientation and period vector (replication vector) of the periodic moiré image bands (Fig. 3b). In addition, the model is also able to predict the translation of the moiré image bands when translating the revealing layer on top of the base layer.

We are also interested in creating moiré images with curvilinear base and revealing layers, each one obtained by a non-linear geometric transformation of its corresponding rectilinear ${ }^{1}$ periodic layer. The challenge resides in the development of a mathematical model characterizing the geometric transformation of the moire image between original and transformed spaces. Having mastered this transformation, we may then produce rectilinear moiré shapes despite the fact that both the base layer and the revealing layer are curvilinear.

A last challenge resides in the exploitation of the models to create interesting visual effects, such as creating within the same design moiré patterns which move along different orientations and at different speeds when applying a translation to the revealing layer.

In section 2, we describe related work, and in particular the concept of indicial equations useful for characterizing the layout of moiré fringes. In section 3, we deduce, by relying on geometric considerations, the linear transformation between rectilinear base layer space and rectilinear moiré image space. In section 4 , we describe various design options for creating rectilinear moiré images. In section 5, we synthesize curvilinear base and revealing layers by applying geometric transformations to the original layers. We then derive the geometric transformation between the original rectilinear moiré image and the transformed moiré image. In section 6 , we benefit from the capabilities offered by band moirés to create various design effects. In section 7 , we draw the conclusions.

\section{Related work}

The geometry of moiré fringes, i.e. their orientation and period has been studied by analyzing the properties of superposed layers, their periods and their angles [Tollenaar 1945], [Nishijima and Oster 1964]. One may obtain both the geometric properties (period, orientation) and the intensity profile of the moiré fringes by considering in the Fourier domain the superposition of two gratings as the convolution of their respective spectra [Bryngdahl 1974], [Amidror 2000, Chapters 2 and 4].

A further method of analyzing moiré fringes relies on the indicial equations of the families of lines composing the base and revealing $^{2}$ layer line gratings. The moiré fringes formed by the superposition of these indexed line families form a new family of indexed lines whose equation is deduced from the equation of the base and revealing layer line families [Oster, Wasserman and Zwerling 1964], [Amidror 2000, Section 11.2]. Fig. 4 shows the oblique base layer lines with indices $n=-1,0,1,2,3, . .$, the transparent horizontal revealing layer lines with indices $m=0,1,2,3,4, .$. and the moiré fringe lines (in red) with indices $\mathrm{k}=1,0,-1,-2 \ldots$.

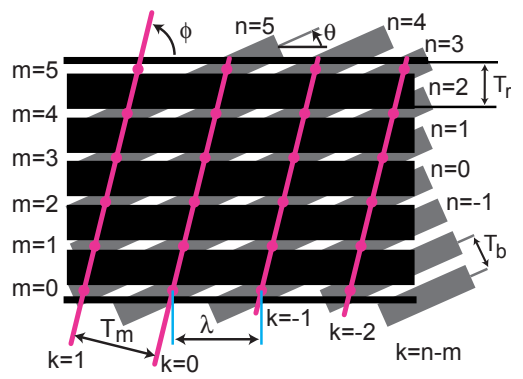

Fig. 4. Superposition of two line gratings, the resulting moiré fringe lines (red) and their corresponding line indices.

1. A layer is rectilinear if its band borders or respectively its revealing lines are straight lines.

2. The revealing layer is always the second layer which is superposed on top of a first layer. 
The moiré fringe lines comprise dark moiré lines connecting the intersections of dark oblique and transparent horizontal revealing layer lines (red lines in Fig. 4). Each dark moiré line can be characterized by an index

$$
k=n-m
$$

With the equations describing the family of oblique base lines

$$
y=\tan \theta \cdot x+n \cdot \lambda \cdot \tan \theta
$$

and the family of horizontal revealing lines

$$
y=m \cdot T_{r}
$$

and by expressing indices $n$ and $m$ as functions of $x$ and $y$, we can, thanks to equation (1), deduce the equation describing the family of moiré fringe lines

$$
y=x \cdot \frac{T_{r} \cdot \tan \theta}{T_{r}-\lambda \cdot \tan \theta}+k \cdot \frac{T_{r} \cdot \lambda \cdot \tan \theta}{T_{r}-\lambda \cdot \tan \theta}
$$

Equation (4) fully describes the family of subtractive ${ }^{1}$ moire fringe lines: the moire fringe lines orientation is given by the slope of the line family and their period can be deduced from the vertical spacing between two successive lines.

In section 5, we will reuse indicial equation (4) in order to deduce the geometric transformation of the moiré images whose base and revealing layers are geometrically transformed. Achieving this goal by relying on indicial equations seems to be simpler than by relying on Fourier theory [Amidror 2000, Chap. 10]. With Fourier theory, one needs non-trivial extensions to describe curvilinear repetitive structures in Fourier space [Amidror 1997, 1998].

Thanks to a variational approach, Lebanon and Bruckstein [2001] compute from a desired moiré fringe lines function $g(x, y)=k$ the corresponding base layer and revealing layer line grating functions $\psi(x, y)=n$ and $\phi(x, y)=m$ so as to obtain an approximate maximally visible subtractive moiré fringe lines function $\bar{g}(x, y)=\psi(x, y)-\phi(x, y)$. This work only considers traditional periodic profile moiré fringe lines and is therefore not directly related to band moiré images. However, both studies share the aim of deducing a base layer and a revealing layer whose superposition produces a desired moiré pattern.

Joe Huck [2003], a prepress specialist, became aware of band moiré images when creating designs incorporating moiré shapes floating within different perceived depth planes thanks to parallax effects. However, he neither provided a general-purpose framework for predicting the geometry of band moiré images as a function of base and revealing layer layouts, nor did he consider geometric transformations of base and revealing layers.

For the sake of completeness, let us also mention the phase based approach, where a latent image embedded within a base layer is revealed by placing a revealing layer such as lenticular lenses at a particular phase and orientation on top of the base layer [McGrew 1995]. In contrast to moiré based approaches, phase based techniques cannot produce smoothly varying dynamic patterns upon translation or rotation of the revealing layer.

\section{The geometry of periodic band moiré images}

The geometric model we are describing relies on the assumption that the revealing line grating is made of transparent straight lines with a small relative aperture, i.e. the revealing line grating can be assimilated to a grating of sampling lines.

Let us analyze how the revealing line grating (red lines in Fig. 5)

1. Other moiré fringes such as additive ones exist, but are less visible [Oster, Wasserman \& Zwerling 1964] samples the underlying base layer formed by replications of oblique base band $B_{0}$, yielding base bands $B_{-1}, B_{1}, B_{2}, B_{3}, B_{4}$ (Fig. 5).

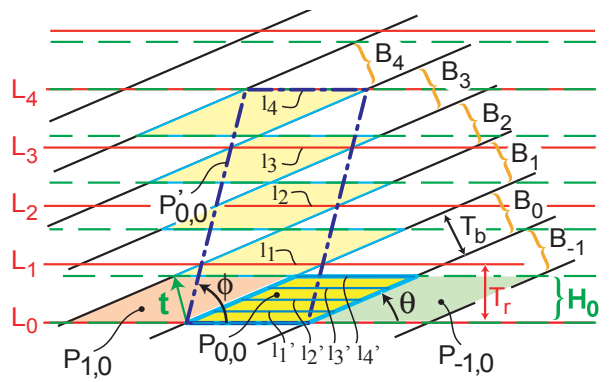

Fig. 5. Superposition of base bands and revealing line grating

Base bands are replicated with replication vector $\boldsymbol{t}$. Oblique base bands $B_{1}, B_{2}, B_{3}, B_{4}$ are by construction exact replicates of base band $B_{0}$. The yellow parallelograms located respectively in oblique bands $B_{1}, B_{2}, B_{3}, B_{4}$ (Fig. 5) are therefore exact replicates of the base parallelogram $P_{0,0}$ located in band $B_{0}$.

The revealing line grating (red revealing lines $L_{0}, L_{1}, L_{2}, L_{3}, L_{4}$, Fig. 5), superposed on top of the base layer samples the replicated base bands and produces a moiré image (Fig. 3b). The intersections of the revealing lines (sampling lines) with replicates of base band parallelogram $P_{0,0}$, i.e. the sampled line segments $l_{1}, l_{2}, l_{3}, l_{4}$ are identical to the sampled line segments $l_{1}, l_{2}, l_{3}$, $l_{4}$ ' within base band parallelogram $P_{0,0}$. We therefore observe a linear transformation mapping base band parallelogram $P_{0,0}$ to moiré parallelogram $P_{0,0}$ '. We will show that this linear transformation depends on the base band replication vector $t$ and on the revealing line grating period $T_{r}$

The observed linear transformation also applies to all other base band parallelograms (e.g. $P_{1,0}, P_{-1,0}$ ) which are horizontal neighbors of base band parallelogram $P_{0,0}$ and which form a horizontal band $H_{0}$ parallel to the revealing lines (Fig. 5). Successive horizontal bands are labelled $H_{0}, H_{1}, H_{2}, H_{3}$ (Fig. 6). Base band parallelograms at the intersection of oblique base band $u$ and horizontal band $v$ are denominated $P_{u, v}$.

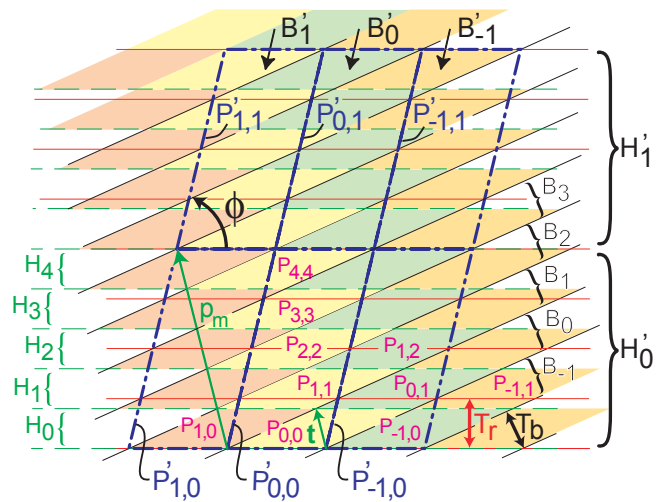

Fig. 6. Replicated horizontal bands $H_{i}$, corresponding replicated horizontal moiré bands $H_{i}$, replicated oblique base bands $B_{j}$ and corresponding replicated oblique moiré bands $B_{j}$.

Neighbor parallelograms within a horizontal band $\left[. ., P_{1,0}, P_{0,0}, P_{\text {. }}\right.$ $1,0, .$.$] are mapped to horizontal moiré neighbor parallelograms$ $\left[. ., P_{1,0}, P_{0,0}, P_{-1,0}, \ldots\right]$. Neighbor parallelograms within an oblique base band $\left[. ., P_{0,0}, P_{0,1}, ..\right]$ are mapped to oblique moiré neighbor parallelograms $\left[. ., P_{0,0}, P_{0,1}, . ..\right]$. Therefore, horizontal base bands $H_{0}, H_{1}$ are mapped onto horizontal moiré bands $H_{0}$, $H_{1}$ ' and oblique base bands $B_{0}, B_{1}$ are mapped onto oblique moiré 
bands $B_{0}$ ', $B_{1}$ '(Fig. 6).

Since base band parallelograms $P_{i, i}$ are replicates, corresponding moiré parallelograms $P_{i, i}$ are also replicates ${ }^{1}$. When moving the revealing line grating down with a vertical translation of one period $T_{r}$, the moiré parallelograms $P_{u, v}$ ' move to the position of the moiré parallelograms $P_{u+1, v+1}$ ' (e.g. in Fig. 6, parallelogram $P_{0,0}$ ' moves to the position of parallelogram $P_{1,1}$ ').

Let us establish the parameters of the linear transformation mapping base band parallelograms to moiré parallelograms. Consider the linear mapping of base band parallelogram $P_{0,0}$ into moiré parallelogram $P_{0,0}$ ' (Fig. 7). Points $A$ and $B$ of the base band parallelogram remain fixpoints. Since line segment $l_{1}$ ' is mapped into line segment $l_{1}$, point $G$ of the base band parallelogram $P_{0,0}$ is mapped into point $H$ of the moiré parallelogram $P_{0,0}$ '.

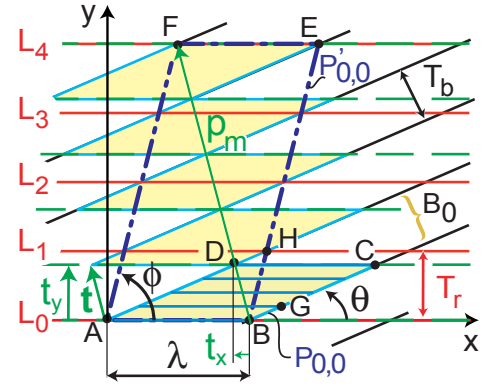

Useful relations:
$\begin{aligned} & \lambda=\left(t_{y} / \tan \theta\right)-t_{x} \\ & \lambda=T_{b} / \sin \theta\end{aligned}$

Fig. 7. Enlarged view of base band parallelogram $P_{0,0}$ and moiré parallelogram $P_{0,0}$ '

The coordinates of point $H$ are given by the intersection of revealing line $L_{1}$ and the upper boundary of oblique base band $B_{0}$. One obtains the coordinates of point $G$ by subtracting from the coordinates of point $H$ the replication vector $\boldsymbol{t}=\left(t_{x}, t_{y}\right)$. We obtain

$$
\begin{aligned}
& H=\left(T_{r} / \tan \theta, T_{r}\right) \quad \text { and } \\
& G=\left(T_{r} / \tan \theta-t_{x}, T_{r}-t_{y}\right)
\end{aligned}
$$

With $\mathrm{B}$ as fixpoint, i.e. $(\lambda, 0)->(\lambda, 0)$, and with $\mathrm{G}->\mathrm{H}$, we obtain the linear transformation mapping base band parallelograms to moiré parallelograms

$$
\left[\begin{array}{l}
x^{\prime} \\
y^{\prime}
\end{array}\right]=\left[\begin{array}{ll}
p & q \\
r & s
\end{array}\right]\left[\begin{array}{l}
x \\
y
\end{array}\right]=\left[\begin{array}{cc}
1 & \frac{t_{x}}{T_{r}-t_{y}} \\
0 & \frac{T_{r}}{T_{r}-t_{y}}
\end{array}\right]\left[\begin{array}{l}
x \\
y
\end{array}\right]
$$

Interestingly, with a constant replication vector $\boldsymbol{t}$, the linear transformation parameters remain constant when modifying angle $\theta$ between the base band and the revealing line grating. However, the orientation $\phi$ of the moiré parallelogram depends on $\theta$. The moiré parallelogram angle $\phi$ can be derived from line segment $\overline{\mathrm{BH}}$, where point $\mathrm{B}$ has the coordinates $(\lambda, 0)$ and where $\lambda=\left(t_{y} / \tan \theta\right)-t_{x}$. With point $\mathrm{H}$ given by Eqs. (5), we obtain for the moiré parallelogram orientation $\phi$

$$
\tan \phi=\frac{T_{r}}{\frac{T_{r}}{\tan \theta}-\lambda}
$$

One can easily verify that indeed, the moire parallelogram slope obtained by the proposed linear transformation between base layer and moiré layer is identical to the slope of the moiré fringe lines

1. Up to a sampling phase difference which is due to the fact that the relative phase of the sampling line may not be the same from one horizontal moiré band to the next. described by their indicial equation (4). This can be explained by considering that moiré fringe lines are a special case of band moiré images. If we replace the oblique base band layer with a line grating of the same orientation, period and phase, we obtain within the oblique moiré parallelogram bands the corresponding moiré fringe lines.

Expressed as a function of its oblique base band width $T_{b}$, with $\lambda=T_{b} / \sin \theta$, the moiré parallelogram orientation

$$
\tan \phi=\frac{T_{r} \cdot \sin \theta}{T_{r} \cdot \cos \theta-T_{b}}
$$

is identical to the familiar moire line orientation formula developed by Tollenaar [1945] according to geometric considerations.

Since both oblique and horizontal moiré parallelogram bands are replicates (Fig. 6), let us deduce the moiré band replication vector $\boldsymbol{p}_{m}$. Base bands are replicated by replication vector $\boldsymbol{t}=\left(t_{x}, t_{y}\right)$. Since there is a linear mapping between base band parallelogram $P_{0,0}$ and moiré parallelogram $P_{0,0}$, whose diagonal is the moiré band replication vector $\boldsymbol{p}_{m}$ (Fig. 7), by mapping point $\left(t_{x}, t_{y}\right)$ according to the linear transformation given by the system of equations (6), we obtain replication vector $\boldsymbol{p}_{m}$

$$
\boldsymbol{p}_{m}=\left(t_{x}+t_{y} \cdot \frac{t_{x}}{T_{r}-t_{y}}, t_{y} \cdot \frac{T_{r}}{T_{r}-t_{y}}\right)=\frac{T_{r}}{T_{r}-t_{y}} \cdot \boldsymbol{t}
$$

The orientation of replication vector $\boldsymbol{p}_{m}$ gives the angle along which the moiré parallelogram bands, i.e. the moiré image travels when displacing the horizontal revealing layer on top of the base layer. This moiré band replication vector is independent of the oblique base band orientation, i.e. one may, for the same base band replication vector $\boldsymbol{t}=\left(t_{x}, t_{y}\right)$ conceive different oblique base bands yielding the same moire band replication vector. However, differently oriented oblique base bands will yield differently oriented oblique moiré bands, which nevertheless will be replicated according to the same replication vector $\boldsymbol{p}_{m}$.

Again, it is possible to verify that, in the special case when the oblique base band layer is replaced by a line grating having the same geometric layout, the moiré bands become moiré fringe lines and their respective period $T_{m}$ (distance between two moiré fringe lines, see Fig. 4) can be deduced from moiré band replication vector $\boldsymbol{p}_{m}$. Period $T_{m}$ is the length of replication vector $\boldsymbol{p}_{m}$ projected onto a unit vector $\boldsymbol{n}$ perpendicular to the moiré parallelogram lines of orientation $\phi$ (Fig. 7, Eq. 7), i.e. $T_{m}=\boldsymbol{n} \cdot \boldsymbol{p}_{m}$. With $t_{x}=\left(t_{y} / \tan \theta\right)-$ $\left(T_{b} / \sin \theta\right)$, we obtain the well known formula for the moire fringe line period

$$
T_{m}=\frac{T_{b} \cdot T_{r}}{\sqrt{T_{b}^{2}+T_{r}^{2}-2 \cdot T_{b} \cdot T_{r} \cdot \cos \theta}}
$$

which was first developed by Righi [1887], then by Tollenaar [1945] and later by Nishijima and Oster [1964] according to geometric considerations.

\section{Synthesis of straight band moiré images}

By considering the revealing line grating as a sampling line array, we were able to define the linear transformation between the base layer and the moiré image (section 3 ). The base layer is formed by an image laid out within a single base band replicated with vector $t$ so as to cover the complete base layer space.

In order to better understand the various moiré image design alternatives, let us try to create a text message within the base layer according to different layout alternatives. 
One may for example conceive a flattened microtext (or graphical elements) running along the oblique base bands at orientation $\theta$ (Fig. 8). In the moiré image, the corresponding linearly transformed enlarged microtext will then run along the oblique moiré bands at orientation $\phi$.

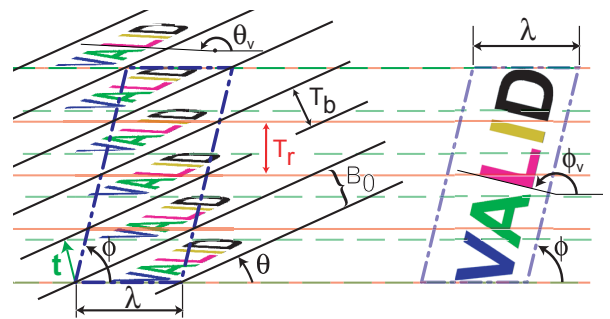

Fig. 8. Microtext running within oblique base bands

The microtext's vertical orientation can also be chosen. With equation (7) expressing the relationship between orientations within the base band layer and orientations within the moiré image layer, one may compute the vertical bar orientation (angle $\theta_{\mathrm{v}}$ of the vertical bar of letter "L" in Fig. 8) of the microtext which in the moiré image yields an upright text, i.e. a text whose vertical orientation (angle $\phi_{\mathrm{v}}=\phi+90^{\circ}$ ) is perpendicular to its baseline. We first express $\theta_{v}$ as a function of $\phi_{v}$, replace $\phi_{v}$ by $\phi+90^{\circ}$, and finally express $\phi$ as a function of $\theta$. We obtain the microtext's vertical orientation $\theta_{v}$ yielding an upright text in the moiré image

$$
\cot \theta_{v}=\frac{1}{\frac{\lambda}{T_{r}}-\cot \theta}+\frac{\lambda}{T_{r}}
$$

Clearly, the orientation of the revealed moiré text baseline (angle $\phi)$ is given by the orientation of the oblique band (angle $\theta$ ). The height of the characters depends on the oblique base band base $\lambda$ or, equivalently, on its width $T_{b}$. The moiré band repetition vector $\boldsymbol{p}_{m}$ which defines how the moiré image is translated when moving the revealing layer up and down, depends according to Eq. (9) on replication vector $\boldsymbol{t}=\left(t_{x}, t_{y}\right)$. Once the moiré text baseline orientation $\theta$ and oblique band base $\lambda$ have been chosen, one may still modify replication vector $\boldsymbol{t}$ by moving its head along the oblique base band border. By choosing a vertical component $t_{y}$ closer to $T_{r}$, the vertical enlargement factor $s$ becomes larger according to Eq. (6) and the moiré image becomes higher, i.e. the moiré text becomes more elongated.

Alternatively, instead of designing the microtext within the oblique base bands, one may design microtext within a horizontal base band (Fig. 9) whose height is given by the vertical component $t_{y}$ of base band replication vector $\boldsymbol{t}=\left(t_{x}, t_{y}\right)$. By replicating this horizontal base band with replication vector $t$, we populate the base layer.

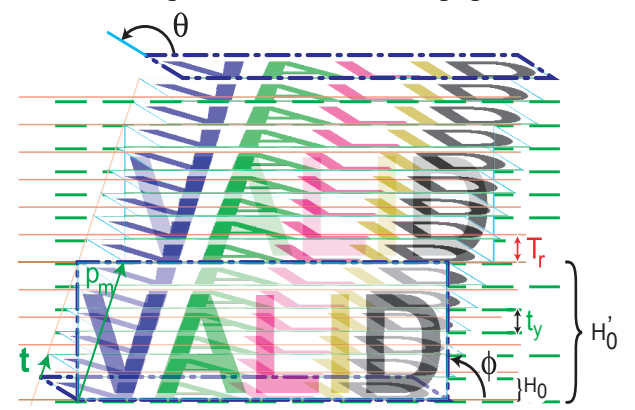

Fig. 9. Microtext laid out within a horizontal base and the corresponding moiré text image.

The vertical orientation $\theta$ of the microtext can be freely chosen. It defines the layout of the corresponding oblique bands and there- fore, the vertical orientation $\phi$ of the revealed moire text image (linearly transformed enlarged microtext). The selected replication vector $\boldsymbol{t}$ defines the vertical size of the moiré band $H_{0}$ ' (see also Fig. 6), i.e. the vertical extension of the revealed moiré text image and its displacement direction $\boldsymbol{p}_{m}$ when the revealing layer moves on top of the base layer (Eq. 9).

The choice of the revealing line period $T_{r}$ depends on the base layer resolution. Generally the period $T_{r}$ of the revealing line grating is between $5 \%$ to $10 \%$ smaller or larger than the horizontal base band layer width $t_{y}$. Considering equation (6), factor $s=T_{r} /\left(T_{r}-t_{y}\right)$ defines the vertical enlargement between the flattened image located within a horizontal base band $H_{0}$ and the moire image located within the corresponding moiré horizontal band $H_{0}$ '. The horizontal base band width $t_{y}$ should offer enough resolution to sample the vertically compressed text or graphical design (vertical compression factor: $s$ ). At 1200 dpi, a horizontal base band width of half a millimeter corresponds to 24 pixels. This is enough for displaying text or line graphics. Therefore, at a resolution between $1200 \mathrm{dpi}$ and $600 \mathrm{dpi}$, we generally select a revealing line grating period between one half to one millimeter. The aperture of the revealing layer, i.e. the width of its transparent lines is between $10 \%$ to $15 \%$ of its period $T_{r}$. There is a trade-off between a small aperture leading to a sharp but dark moiré image and a large aperture leading to a highlight but blurred ${ }^{1}$ moiré image.

The creation of moiré images does not necessarily need a sophisticated computer-aided design system. Let us illustrate the moiré image creation procedure in the case of a microtext laid out within a horizontal base band.

One may simply start by defining the period $T_{r}$ of the revealing layer. Then one creates the desired "moiré" image within a horizontal parallelogram whose sides define the orientation $\phi$ of the oblique moiré band borders $B_{i}^{\prime}$ (see Figs. 6,8,9). The horizontal parallelogram height defines the vertical size of the moiré band $H_{0}$, i.e. the vertical component of replication vector $\boldsymbol{p}_{m}$ and therefore according to Eq. (9) the vertical component $t_{y}$ of replication vector $\boldsymbol{t}$. One then needs to linearly transform the horizontal moiré image parallelogram in order to fit it within a horizontal band of height $t_{y}$. This "flattening" operation has one degree of freedom, i.e. point $F$ (Fig. 7) may be freely mapped to a point $D$ located at the top border of the horizontal base band. The mapping between point $F$ and point $D$ yields the value of $\lambda$ and the horizontal component $t_{x}$ of replication vector $t$. By modifying the position of point $D$ along the top border of the horizontal base band, one modifies the horizontal component $t_{x}$ of vector $\boldsymbol{t}$ and therefore the orientation $\boldsymbol{p}_{m}$ along which the moiré parallelogram moves when translating the revealing layer on top of the base layer (Figs. 6, 9). The creation of an image within a "moiré" parallelogram, its linear transformation in order to fit it within a horizontal base band and the replication of that base band can be carried out with a standard software package such as Adobe Photoshop or Illustrator.

The moiré design procedure described above may serve as a guideline for developing an interactive moiré image design system. However further experience is needed in order to understand how designers will create moiré designs, preview them and modify them interactively. Further issues of interest are the embedding of moiré images within designs incorporating other elements and the incorporation of revealing layers attached to the base layer so as to offer only one degree of freedom, e.g. translation along one axis or rotation around one point.

1. The analysis about how the revealing layer blurs the underlying base band image is outside the scope of the present contribution, see [Amidror 2000, Chapter 4]. 


\section{Geometrically transformed base and revealing layers}

Starting with a periodic rectilinear base band layer and a periodic rectilinear straight line revealing grating, one may apply to them either the same or different non-linear geometric transformations. We expect that the curved moiré image we obtain is a geometric transformation of the original band moiré image obtained by superposing the periodic rectilinear base band and revealing line gratings. We would like to find the geometric transformation which gives the mapping between the resulting curved moiré image and the original periodic band moiré image.

This geometric transformation should yield, for the special case of a base layer made of a curved line grating, the mapping obtained by Amidror [2000, section 10.9]. He performed a detailed analysis of the superposition of curved line gratings in Fourier space, and has shown that in the case of two superposed curved line gratings, the corresponding moire transformation is the difference of the geometric transformations of the normalized individual line gratings.

The key element for deriving the transformation between curvilinear and original moiré images is the determination of parameters within the moiré image, which remain invariant under layer transformations, i.e. geometric transformations of base and revealing layers.

One parameter remaining invariant is the index $k$ of the oblique moiré band borders (Figs. 6, 10a), which correspond to the subtractive moiré lines (Fig. 4). The curved (transformed) moiré band borders are given by the intersections of curved base band borders and curved revealing lines (Fig. 10b). We may according to the indicial approach (Fig. 10a) describe any point $P$ within the base layer space or respectively within the revealing layer space as being located on one oblique base band line of index $n$ ( $n$ being a real number) or respectively on one revealing grating line of index $m$ ( $m$ being a real number). Clearly, under a geometric transformation of their respective layers, indices $n$ and $m$ remain invariant. The intersection between an oblique base band line of index $n$ and a revealing grating line of index $m$ yields a moiré image line of index $k=n-m$ ( $k$ being a real number), both before applying the geometric transformations (Fig. 10a) and after applying these transformations (Fig. 10b).
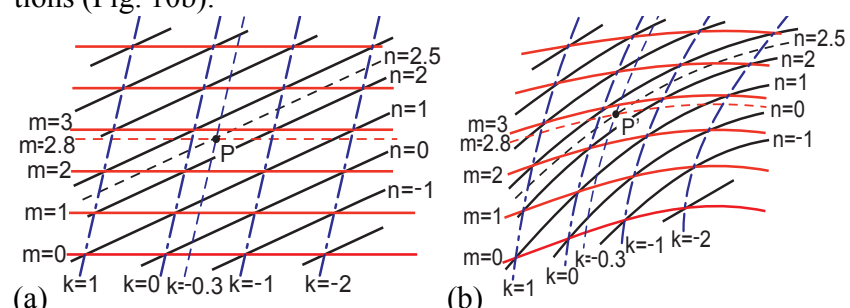

Fig. 10. Indices of oblique base band borders $n$, of revealing lines $m$, and of the corresponding moiré band border lines $k$, (a) before and (b) after applying the geometric transformations.

Eq. (4) gives the family of moiré image lines (Figs. 4 and 10a) parallel to the oblique moiré band borders before applying the geometric transformations. Let us define the geometric transformation between transformed base layer space $\left(x_{t}, y_{t}\right)$ and original base layer space $(x, y)$ by

$$
x=h_{1}\left(x_{t}, y_{t}\right) ; \quad y=h_{2}\left(x_{t}, y_{t}\right)
$$

and the geometric transformation between transformed revealing layer space $\left(x_{t}, y_{t}\right)$ and original revealing layer space $(x, y)$ by ${ }^{1}$

$$
y=g_{2}\left(x_{t}, y_{t}\right)
$$

We can insert these geometric transformations into respectively equation (2) describing the family of oblique base band lines and equation (3) describing the family of revealing lines

$$
n=\frac{h_{2}\left(x_{t}, y_{t}\right)-h_{1}\left(x_{t}, y_{t}\right) \cdot \tan \theta}{\lambda \cdot \tan \theta} ; \quad m=\frac{g_{2}\left(x_{t}, y_{t}\right)}{T_{r}}
$$

With equation (1), we obtain the implicit equation of the moiré image lines in the transformed space according to their indices $k$

$$
k=n-m=\frac{h_{2}\left(x_{t}, y_{t}\right) \cdot T_{r}-h_{1}\left(x_{t}, y_{t}\right) \cdot T_{r} \cdot \tan \theta-g_{2}\left(x_{t}, y_{t}\right) \cdot \lambda \cdot \tan \theta}{\lambda \cdot T_{r} \cdot \tan \theta}
$$

Since the moire line indices $k$ are the same in the original (Eq. 4) and in the transformed spaces (Eq. 15), by equating them, we obtain an implicit equation establishing a relationship between transformed and original moiré space coordinates having the form $F_{k}\left(x_{t}, y_{t}, x, y\right)=0$. We obtain

$$
\begin{aligned}
& F_{k}\left(x_{t}, y_{t}, x, y\right)=h_{2}\left(x_{t}, y_{t}\right) \cdot T_{r}-h_{1}\left(x_{t}, y_{t}\right) \cdot T_{r} \cdot \tan \theta \\
& -g_{2}\left(x_{t}, y_{t}\right) \cdot \lambda \cdot \tan \theta+x \cdot T_{r} \cdot \tan \theta+y \cdot\left(\lambda \cdot \tan \theta-T_{r}\right)=0
\end{aligned}
$$

To completely specify the mapping between each point of the transformed moiré space and each point of the original moiré space, we need an additional implicit equation relating transformed and original moiré image layer coordinates.

We observe that replicating oblique base bands with the replication vector $\boldsymbol{t}$ is identical to replicating horizontal base bands with replication vector $\boldsymbol{t}$ (Fig. 6). We can therefore concentrate our attention on the moiré produced by superposing the horizontal revealing line grating and the horizontal base bands (Fig. 11).

Clearly, base band parallelogram $\mathrm{P}_{\lambda t}$ with base $\lambda$ and with replication vectors $\boldsymbol{t}$ as parallelogram sides is mapped by the linear transformation (Eq. 6) into the moiré parallelogram $\mathrm{P}_{\lambda t}$ ' having the same base $\lambda$ and parallelogram sides given by moiré band replication vector $\boldsymbol{p}_{m}$ (Fig. 11). Successive vertically adjacent replicates of moiré parallelogram $\mathrm{P}_{\lambda t}$ ' are mapped by the linear transformation into identical replicas of the base band parallelogram $\mathrm{P}_{\lambda t}$. Therefore, within the moiré image, each infinite line of orientation $\boldsymbol{p}_{m}$, called $d$-line is only composed of replicates of a single line segment $d_{b}$ parallel to $t$ within the base band. This is true, independently of the value of the revealing grating period $T_{r}$.

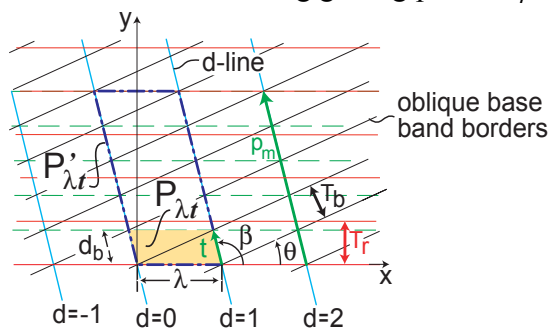

Fig. 11. Base band parallelogram of orientation $\boldsymbol{t}$ linearly transformed into a moiré parallelogram of the same orientation

With a given horizontal base band and a base band replication vector $\boldsymbol{t}$ forming an angle $\beta$ with the horizontal, we can generate an infinite number of oblique base band layouts by rotating them around vector $t$. The smaller the difference between angles $\theta$ and $\beta$, the smaller the base segment $\lambda$ (Fig. 11). Oblique bands oriented according to vector $\boldsymbol{t}$, i.e. with an angle $\theta=\beta$, become infinitely thin. At this orientation, an infinite number of oblique base band

\footnotetext{
1. Any superposition of original base and revealing layers can be rotated so as to obtain a horizontal revealing layer, whose line family equation depends only on the y-coordinate. The transformation from transformed space to original space comprises therefore only the single function $y=g_{2}\left(x_{t}, y_{t}\right)$.
} 
borders fall into a single d-line. This d-line therefore becomes the moiré line located at the intersections between oblique base band borders and revealing lines. This moiré line (d-line) remains identical when the base band borders are intersected with a geometrically transformed revealing line layer. Therefore, d-lines within the moiré image space remain invariant under geometric transformation of the revealing layer. In Fig. 13 for example, when applying to the revealing layer a rotation, a translation or any other transformation, points of the original moiré image move only along their respective d-lines.

Under geometric transformation of the base layer, straight d-lines are possibly transformed into curved d-lines. In the moiré image space, a point located on a straight d-line will remain, after application of a geometric transformation to the revealing layer and of a (generally different) geometric transformation to the base layer, on the corresponding transformed curved d-line.

By numbering the d-lines according to d-parallelogram borders (Fig. 11), we can associate every point within the moiré image to a d-line index (real number). Since the d-line indices are the same in the original and in the transformed moiré image, we can equate them and establish an implicit equation of the form $F_{d}\left(x_{t}, y_{t}, x, y\right)=0$.

The d-line family equations become in the original space

$$
y=x \cdot \tan \beta-d \cdot \lambda \cdot \tan \beta
$$

and in the transformed space, by replacing $x$ and $y$ with respectively $h_{1}$ and $h_{2}$

$$
h_{2}\left(x_{t}, y_{t}\right)=h_{1}\left(x_{t}, y_{t}\right) \cdot \tan \beta-d \cdot \lambda \cdot \tan \beta
$$

where $\beta$ is the angle of replication vector $\boldsymbol{t}$ with the horizontal and where $d$ is the d-line index. If we extract the d-line index $d$ from equation (17) and also from equation (18), by equating them, we obtain the following implicit equation

$F_{d}\left(x_{t}, y_{t}, x, y\right)=h_{2}\left(x_{t}, y_{t}\right)-h_{1}\left(x_{t}, y_{t}\right) \cdot \tan \beta+x \cdot \tan \beta-y=0$

We can now solve for $x$ and $y$ the equation system formed by $F_{k}\left(x_{t}, y_{t}, x, y\right)=0$ (Eq. 16) and $F_{d}\left(x_{t}, y_{t}, x, y\right)=0$ (Eq. 19) and obtain, by replacing $\lambda$ in equation (16)

$$
\lambda=t_{y} \cot \theta-t_{x}=t_{y}(\cot \theta-\cot \beta)
$$

the geometric transformation $\left(m_{1}\left(x_{t}, y_{t}\right), m_{2}\left(x_{t}, y_{t}\right)\right)$ of the moiré image from transformed moiré space to original moiré space

$$
\begin{aligned}
& x=m_{1}\left(x_{t}, y_{t}\right)=h_{1}\left(x_{t}, y_{t}\right)+\left(h_{2}\left(x_{t}, y_{t}\right)-g_{2}\left(x_{t}, y_{t}\right)\right) \cdot \frac{t_{y} \cdot \cot \beta}{T_{r}-t_{y}} \\
& y=m_{2}\left(x_{t}, y_{t}\right)=h_{2}\left(x_{t}, y_{t}\right) \cdot \frac{T_{r}}{T_{r}-t_{y}}-g_{2}\left(x_{t}, y_{t}\right) \cdot \frac{t_{y}}{T_{r}-t_{y}}
\end{aligned}
$$

Equations (21) confirm that the geometric transformation of the moiré image (moiré transformation) is independent of the oblique base band orientation. Relevant parameters are the revealing layer line period $T_{r}$, the horizontal base band width $t_{y}$ and angle $\beta$ of replication vector $\boldsymbol{t}$ (or equivalently, the horizontal component $t_{x}=$ $t_{y} \cot \beta$ of replication vector $\boldsymbol{t}$ ).

Equations (21) show that when the geometric transformations of base layer and revealing layer are identical i.e. $h_{2}\left(x_{t}, y_{t}\right)=g_{2}\left(x_{t}, y_{t}\right)$, the moire transformation is identical to the transformation of the base layer, i.e. $m_{1}\left(x_{t}, y_{t}\right)=h_{1}\left(x_{t}, y_{t}\right)$ and $m_{2}\left(x_{t}, y_{t}\right)=h_{2}\left(x_{t}, y_{t}\right)$.

The moiré transformation (Eq. 21) resulting from a geometric transformation of the base layer by $x=h_{1}\left(x_{t}, y_{t}\right), y=h_{2}\left(x_{t}, y_{t}\right)$ and of the revealing line grating layer by $y=g_{2}\left(x_{t}, y_{t}\right)$ is consistent (see Appendix) with the result of Amidror [2000, Section 10.9] when the base band layer is replaced by a line grating of the same geometric layout.

Having obtained the full expression for the induced moiré transfor- mation when transforming base and revealing layers, we can select a given moiré transformation i.e. $m_{1}\left(x_{t}, y_{t}\right)$ and $m_{2}\left(x_{t}, y_{t}\right)$, select one of the two transformations $h_{2}$ or $g_{2}$ and derive the two other transformations by solving equation system (21). The easiest way to proceed is to freely define revealing layer transformation $g_{2}$, and then deduce the base layer transformations $h_{1}$ and $h_{2}$.

$$
\begin{aligned}
& h_{1}\left(x_{t}, y_{t}\right)=\left(g_{2}\left(x_{t}, y_{t}\right)-m_{2}\left(x_{t}, y_{t}\right)\right) \cdot \frac{t_{y} \cdot \cot \beta}{T_{r}}+m_{1}\left(x_{t}, y_{t}\right) \\
& h_{2}\left(x_{t}, y_{t}\right)=g_{2}\left(x_{t}, y_{t}\right) \cdot \frac{t_{y}}{T_{r}}+m_{2}\left(x_{t}, y_{t}\right) \cdot \frac{T_{r}-t_{y}}{T_{r}}
\end{aligned}
$$

In the examples given in section 6 , we freely choose a transformation $g_{2}$, enforce as moiré transformation a simple identity, and are able to generate the same moiré image before and after the layer transformations. We obtain periodic moiré images, despite the fact that both the base layer and the revealing layer are curved, i.e. nonperiodic.

\section{Moiré images: design variations}

Let us present a few design variants making use of the geometric framework developed in the previous sections. We consider first the simple text strings "EPFL", "VALID" and "CARD". Each text string has a specific color, a specific layout and a specific replication vector $\boldsymbol{t}$. All distance values are given in pixels at $2400 \mathrm{dpi}$. "EPFL" is laid out within an oblique band of orientation $\theta=-1.8^{\circ}$, $t_{x}=-15.65, t_{y}=43$. "VALID" and "CARD" are each laid out within a horizontal band, with respective replication vectors $\left(t_{x}=9.64\right.$, $\left.t_{y}=36\right)$ and $\left(t_{x}=11.25, t_{y}=42\right)$ and respective character verticals at orientations $\theta=162.7^{\circ}$ and $\theta=14.92^{\circ}$. The revealing layer has a period $T_{r}=39$. The corresponding base layers superposed with the single revealing layer yield a moiré image composed of 3 differently oriented text pieces travelling up or down along different directions at different relative speeds (Fig. 13).

Let us now apply the knowledge developed in section 5 and create a transformation of the base layer and the revealing layer (Fig. 14) which yields the same moiré image as the moiré image formed by the original layers (Fig. 13), i.e. $m_{1}\left(x_{t}, y_{t}\right)=x_{t}$ and $m_{2}\left(x_{t}, y_{t}\right)=y_{t}$. We define the revealing layer transformation

$$
g_{2}\left(x_{t}, y_{t}\right)=y_{t}+c_{1} \cos \left(2 \pi\left(x_{t}+c_{3}\right) / c_{2}\right)
$$

with $c_{1}, c_{2}$ and $c_{3}$ representing constants and deduce from equations (22) the $2 \mathrm{D}$ transformation to be applied to the base layer, i.e.

$$
\begin{aligned}
& h_{1}\left(x_{t}, y_{t}\right)=x_{t}+c_{1} \cos \left(2 \pi\left(x_{t}+c_{3}\right) / c_{2}\right)\left(t_{y} / T_{r}\right) \cot \beta \\
& h_{2}\left(x_{t}, y_{t}\right)=y_{t}+c_{1} \cos \left(2 \pi\left(x_{t}+c_{3}\right) / c_{2}\right)\left(t_{y} / T_{r}\right)
\end{aligned}
$$

We can move the revealing layer up and down, and the moiré image shapes (Fig. 14) will simply be translated without incurring further deformations. We can verify that such a translation does not modify the resulting moiré image (presently an identity) by inserting into equations (21) the transformations $g_{2}$ (Eq. 23) and $h_{1}, h_{2}$ (Eqs. 24) and by replacing in $g_{2}\left(x_{t}, y_{t}\right)$ coordinate $y_{t}$ by its translated version $y_{t}+\Delta y_{t}$. We obtain

$$
\begin{aligned}
& m_{1}\left(x_{t}, y_{t}\right)=x_{t}-t_{x} \Delta y_{t} /\left(T_{r}-t_{y}\right) \text { and } \\
& m_{2}\left(x_{t}, y_{t}\right)=y_{t}-t_{y} \Delta y_{t} /\left(T_{r}-t_{y}\right),
\end{aligned}
$$

i.e. the original moiré image is simply translated according to vector $\boldsymbol{t}$, scaled by the relative vertical displacement $\Delta y_{t} /\left(T_{r}-t_{y}\right)$. If we apply the same analysis to verify the effect of a horizontal translation of the geometrically transformed revealing layer, we obtain a deformation of the moiré image shape according to a trigonometric function.

Let us now define a revealing layer transformation derived from an egg-crate function

$$
g_{2}\left(x_{t}, y_{t}\right)=y_{t}+c_{1} \cos \left(2 \pi x_{t} / c_{2}\right) \cos \left(2 \pi y_{t} / c_{3}\right)
$$


We obtain for the base layer transformations

$$
\begin{aligned}
& h_{1}\left(x_{t}, y_{t}\right)=x_{t}+c_{1} \cos \left(2 \pi x_{t} / c_{2}\right) \cdot \cos \left(2 \pi y_{t} / c_{3}\right)\left(t_{y} / T_{r}\right) \cot \beta(27) \\
& h_{2}\left(x_{t}, y_{t}\right)=y_{t}+c_{1} \cos \left(2 \pi x_{t} / c_{2}\right) \cdot \cos \left(2 \pi y_{t} / c_{3}\right)\left(t_{y} / T_{r}\right)
\end{aligned}
$$

As shown in Fig. 15, these transformations yield a rectilinear moiré image when the revealing layer is superposed on top of the base layer without any translation. With this set of transformations, both a vertical translation $\Delta y_{t}$ and a horizontal translation $\Delta x_{t}$ of the revealing layer on top of the base layer induce a non-linear transformation of the moiré image. However, since the revealing layer is periodic with period $\left(c_{2}, c_{3}\right)$, the moiré image becomes rectilinear when the translation vector's horizontal component $\Delta x_{t}$ is an integer multiple of the revealing layer's horizontal period $c_{2}$ and its vertical component $\Delta y_{t}$ is an integer multiple of its vertical period $c_{3}$. In the example of Fig. 15, the horizontal and vertical periods are 600 pixels, i.e. $1 / 4$ " at 2400 dpi. The periodic occurrence of the rectilinear moiré image transformation upon translation of the revealing layer may also be verified by inserting into equation (21) the transformations $g_{2}$ (Eq. 26) and $h_{1}, h_{2}$ (Eqs. 27) and by replacing in $g_{2}\left(x_{t}, y_{t}\right)$ coordinates $\left(x_{t}, y_{t}\right)$ by their translated version $\left(y_{t}\right.$ $\left.+\Delta y_{t}, y_{t}+\Delta y_{t}\right)$.

In another example (Fig. 16), we introduce a revealing layer transformation yielding a circular revealing line grating

$$
g_{2}\left(x_{t}, y_{t}\right)=\sqrt{\left(x_{t}-c_{x}\right)^{2}+\left(y_{t}-c_{y}\right)^{2}}
$$

where $c_{x}$ and $c_{y}$ are constants giving the center of the circular grating. In order to obtain a rectilinear moiré image, we define the base layer transformations according to Eq. 22

$$
\begin{aligned}
& h_{1}\left(x_{t}, y_{t}\right)=x_{t}+\left(\sqrt{\left(x_{t}-c_{x}\right)^{2}+\left(y_{t}-c_{y}\right)^{2}}-y_{t}\right) \cdot \frac{t_{y} \cdot \cot \beta}{T_{r}} \\
& h_{2}\left(x_{t}, y_{t}\right)=\sqrt{\left(x_{t}-c_{x}\right)^{2}+\left(y_{t}-c_{y}\right)^{2}} \cdot \frac{t_{y}}{T_{r}}+y_{t} \cdot \frac{T_{r}-t_{y}}{T_{r}}
\end{aligned}
$$

With Fig. 16, the reader may verify the rather surprising fact that the superposition of a strongly curved base band grating and of a perfectly circular revealing line grating yields the original rectilinear moiré image. The center's coordinates are $c_{x}=0$ and $c_{y}=3125$ pixels (at $2400 \mathrm{dpi}, 3125$ pixels correspond to 1.3 " or $33 \mathrm{~mm}$ ).

The examples presented above represent only a few of the many possible transformations that can be applied to the base and revealing layers. Many other transformations can be applied which may produce zone plate gratings (Oster, Wasserman and Zwerling 1964), sinus hyperbolic gratings, etc. With the relationships between the geometric transformations applied to the base and revealing layers and the resulting geometric transformation of the moiré image (Eqs. 21), one may try to compute base and revealing layer transformations in order to obtain a desired moiré image transformation.

We created an appealing design by incorporating base bands with different orientations and repetition vectors into facets of cubes (Fig. 17). Upon translation of the revealing layer, the "EPFL" moiré pattern moves continuously across the different faces of the cubes. Smooth moiré displacement across facet boundaries is achieved by matching the phases of the characters within the adjacent base bands.

In the last example, we created a dynamic design (Fig. 18) inspired by the US flag, where each of the three superposed independent base layers generate upon superposition with the revealing layer a moiré image component moving according to its specific relative speed and orientation.

When two layers have their active parts (foreground) superposed one on top of the other, we either give priority to one layer (e.g. the
USA pattern has priority over the red stripes) or simply superpose the two layers (stars and red stripes). Fig. 12 shows the three base layers and an enlargement of the corresponding base bands (the vertical enlargement factor is twice the horizontal enlargement factor). Please note that when the revealing layer period $T_{r}$ is smaller than the horizontal base band width $t_{y}$, we obtain according to Eq. (6) a negative vertical enlargement factor $s$, i.e. a mirrored moiré image (see "USA" base band pattern in Fig. 12). In such cases, base band patterns need to be vertically mirrored to produce a nonmirrored moiré image.

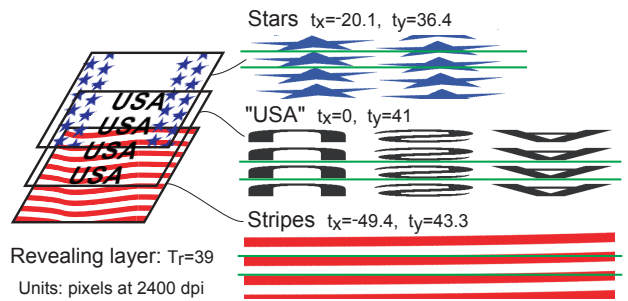

Fig. 12. The different base layers of the design shown in Fig. 18.

\section{Conclusions}

We propose a new powerful way of synthesizing moiré images which allows enough light to be reflected and which enables creating dynamically moving messages incorporating text, symbols, and color elements.

The proposed method for synthesizing moiré images relies on replicated base bands each containing the same 2D base band image. Band moiré images are revealed by a line grating comprising an opaque layer with thin transparent lines. Due to the relatively high amount of light traversing the revealing layer, the induced moiré image is well visible, even in reflective mode.

By considering the formation of the moiré image as a simple line sampling process, we are able to derive the linear transformation between the base layer and the moiré image and to compute the geometric layout of the resulting band moiré image, i.e. its orientation and its replication vector. In the special case where the base band grating is a traditional line grating, our expressions for the orientation and replication vector of the band moiré image directly yield the well known formula for the moiré fringes orientation and period.

Interesting moiré images can be synthesized by applying geometric transformations to both the base and the revealing layers. We therefore develop a mathematical model describing the geometric transformation that a moiré image undergoes when its base and revealing layers are subject to freely chosen generally non-linear geometric transformations. We derive the moiré transformation by searching for parameters remaining invariant under base and revealing layer transformations. These parameters are the indices of the moiré band lines and the indices of the d-lines parallel to the base band replication vector. Thanks to this pair of indices, we are able to formulate two implicit equations expressing the dependence between original and transformed moiré coordinates. These two equations lead to an expression for the geometric transformation that a moiré image undergoes when applying geometric transformations to its base and revealing layers.

In the special case where a line grating is laid out according to the same geometric layout as the base layer, our expression for the geometric transformation of the moiré image is consistent with previous results describing the transformation of moiré fringes upon geometric transformations of the superposed line gratings.

By knowing in advance the layout of a moiré image as function of the layouts of the base and revealing layers, we are able to propose 
strategies for synthesizing moiré images. We create dynamic moiré images, with moiré components running up and down along different orientations and at different speeds upon translation of the revealing layer. We also propose a method for deriving layer transformations whose superposition yield periodic moiré images despite the fact that both the base and the revealing layers are curved. Depending on the chosen layer transformation, the resulting moiré image may either remain periodic or not upon vertical translation of the revealing layer. Interesting effects can be achieved by moiré images which slowly deform upon layer transformation. In the future, it might therefore be interesting to establish metrics for moiré image deformation as a function of revealing layer displacement. One may then try, for a given moiré transformation, to find ranges of parameter and coordinate values inducing a predictable visually attractive deformation of the moiré image.

Band moiré images have the potential of offering a large field of applications ranging from striking designs to the protection of documents against counterfeiting. Band moiré images are well suited for protecting security documents, since they enlarge the finest details present within the base bands, details which may be lost by unauthorized reproduction. Band moiré images are also well suited for creating visually enjoyable effects, e.g. dynamic moiré images moving up and down within a shop window illuminated from behind. Within the field of optical art [Oster 1965], band moiré images offer new means of artistic expression.

Since band moiré images are a direct extension of conventional moiré fringes, one may investigate their use in traditional moiré application fields such as mechanical stress analysis or automatic computation of 3D object surfaces by moiré topography [Takasaki 1970].

\section{Acknowledgements}

The authors would like to thank Isaac Amidror for helpful discussions about curvilinear moiré fringes and the anonymous reviewers for their constructive comments. The present research has been partly financed by the Swiss National Science Foundation (project 2000-065167).

\section{Bibliography}

AmidroR, I. 2000. The Theory of the Moiré Phenomenon, Kluwer, 2000

AMIDROR, I. 1994. A generalized Fourier-based method for the analysis of 2D moiré envelope forms in screen superpositions, Journal of Modern Optics, Vol. 41, No. 9, 1837-1862

AMIDROR, I. 1997. Fourier spectrum of radially periodic images, Journal of the Optical Society of America A, Vol. 14, No. 4, April 1997, 816-826.

AmIDROR, I. 1998. The Fourier-spectrum of circular sine and cosine gratings with arbitrary radial phases, Optics Comm., Vol. 149, 127-134

AMIDROR, I. 2002. A new print-based security strategy for the protection of valuable documents and products using moiré intensity profiles, Optical Security and Counterfeit Deterrence Technique IV, Rudolf L. van Renesse, Editor, Proc. SPIE Vol. 4677, 2002, 89-100

Bracewell, R.N. 1986. The Fourier Transform and its Applications, Mc Graw-Hill

BRYNGDAHL, O. 1974. Moiré: Formation and interpretation, Journal of the Optical Society of America, Vol. 64, No. 10, 1287-1294.

Durelli, A.J., PARKs, V.J. 1970. Moiré Analysis of Strain. Prentice-Hall

HucK J. 2003, Mastering Moirés. Investigating Some of the Fascinating Properties of Interference Patterns, http://pages.sbcglobal.net/joehuck

Hutley, M.C., Hunt, R., Stevens, R.F. and SAvander, P. 1994. The Moiré Magnifier, Pure and Applied Optics, Vol. 3, 133-142

Levanon, G. and Bruckstein, A.M. 2001. Variational approach to moiré pattern synthesis, JOSA A, Vol. 18, No. 6, June 2001, 1371-1382.

MCGREW, 1995. Anticounterfeiting method and device utilizing holograms and pseudorandom dot patterns, US patent No. 5,396,559, 1995
MikAmI, O. 1975. New Image Rotation Using Moiré Lenses, Japan Journal of Applied Physics, Vol. 14, No. 7, 1065-1066

Nishijima, Y. and Oster, G. 1964. Moiré Patterns: Their Application to Refractive Index and Refractive Index Gradient Measurements, Journal of the Optical Society of America, Vol. 54, No. 1, 1-5

Oster, G., Wasserman, M., ZwerLing, C. 1964. Theoretical Interpretation of Moiré Patterns. Journal of the Optical Society of America, Vol. 54, No. 2, 169-175

Oster G. 1965. Optical Art, Applied Optics, Vol. 4, No. 11, 1359-1369

Post, D. 1967. Sharpening and Multiplication of Moiré Fringes, Experimental Mechanics, Vol. 7, April 1967, 154-159

Post, D., Han, B., IfuU, P. 1994. High-Sensitivity Moiré: Experimental Analysis for Mechanics and Materials. Springer Verlag

RigHI, A. 1887, Sui fenomeni che si producono colla sovrappposizione di due reticoli e sopra alcune loro applicazioni, Nuovo Cimento Vol. 21, 203-228 (1887) and Vol. 22, 10-34 (1888)

TAKASAKI, H. 1970. Moiré Topography, Applied Optics, Vol. 9, No. 6, $1467-1472$

TollenaAR, D. 1945. Moiré-Interferentieverschijnselen bij rasterdruk, Amsterdam Instituut voor Grafische Technick, Moiré in halftone printing interference phenomena, published in 1964, reprinted in Indebetouw G. Czarnek R. (Eds.). Selected Papers on Optical Moiré and Applications, SPIE Milestone Series, Vol. MS64, SPIE Press, 1992, 618-633

YULE, J.A.C. 1967. Principles of Color Reproduction, Wiley, 1967, Chap13

\section{APPENDIX}

\section{Moiré induced by geometrically transformed line gratings}

Amidror [2000, Chapter 10] analyzed the superposition of curved gratings in Fourier space, and has shown that in the case of two curved gratings, the geometric transformation applied to the induced subtractive moire is the difference of the geometric transformations mapping the curved gratings onto horizontal normalized individual gratings, i.e.

$$
M_{2}\left(x_{t}, y_{t}\right)=H_{2}\left(x_{t}, y_{t}\right)-G_{2}\left(x_{t}, y_{t}\right)
$$

where $y=H_{2}\left(x_{t}, y_{t}\right)$ is the transformation mapping the geometrically transformed base line grating to a normalized base line grating in the original space, $y=G_{2}\left(x_{t}, y_{t}\right)$ is the transformation mapping the geometrically transformed revealing line grating to a normalized revealing line grating in the original space and $y=M_{2}\left(x_{t}, y_{t}\right)$ is the transformation mapping the geometrically transformed moiré line grating to a normalized moiré line grating in the original space.

Let us show that the moiré transformation equation given in equation (21) meets equation (A1) when the horizontal base band layer is replaced by a line grating of the same period $t_{y}$. As usual, the revealing layer consists of a horizontal revealing line grating of period $T_{\mathrm{r}}$.

Let us consider transformations $h_{2}\left(x_{t}, y_{t}\right)$ (respectively $g_{2}\left(x_{t}, y_{t}\right)$, $m_{2}\left(x_{t}, y_{t}\right)$ ) which transform base bands (respectively revealing lines, moiré bands) from transformed space into original space, yielding horizontal base bands (respectively horizontal revealing lines, horizontal moiré bands). Since the normalized gratings have a period of one and since our horizontal base bands, respectively revealing lines and moiré bands have respective periods of $t_{y}, T_{r}$ and $\left(T_{r} t_{y}\right) /$ $\left(T_{r}-t_{y}\right)$, we have the following relationships

$$
\begin{aligned}
& h_{2}\left(x_{t}, y_{t}\right)=t_{y} \cdot H_{2}\left(x_{t}, y_{t}\right) \\
& g_{2}\left(x_{t}, y_{t}\right)=T_{r} \cdot G_{2}\left(x_{t}, y_{t}\right) \\
& m_{2}\left(x_{t}, y_{t}\right)=\left(T_{r} t_{y}\right) /\left(T_{r}-t_{y}\right) \cdot M_{2}\left(x_{t}, y_{t}\right)
\end{aligned}
$$

By inserting equations (A2) into the second equation of (21), we obtain equation (A1). This shows that moiré fringes induced by the superposition of geometrically transformed line gratings are a special case of band moiré images induced by the superposition of geometrically transformed base band and revealing layers. 

the base layer. Then, move slowly the revealing layer along the vertical orientation.

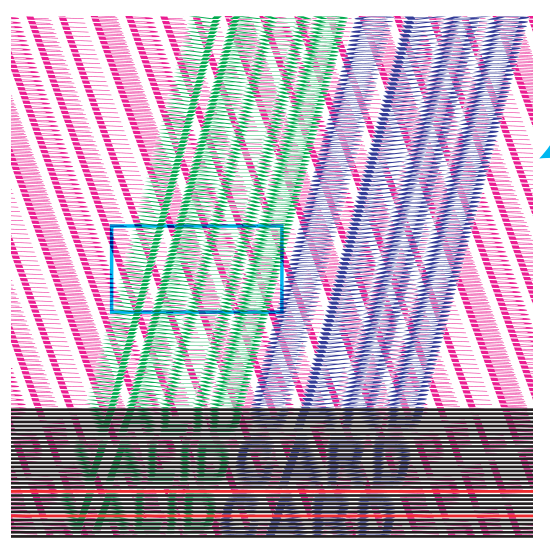

Fig. 13. Rectilinear obliquely oriented "VALID" and "CARD" flying over "EPFL", revealed with a horizontal rectilinear revealing layer.
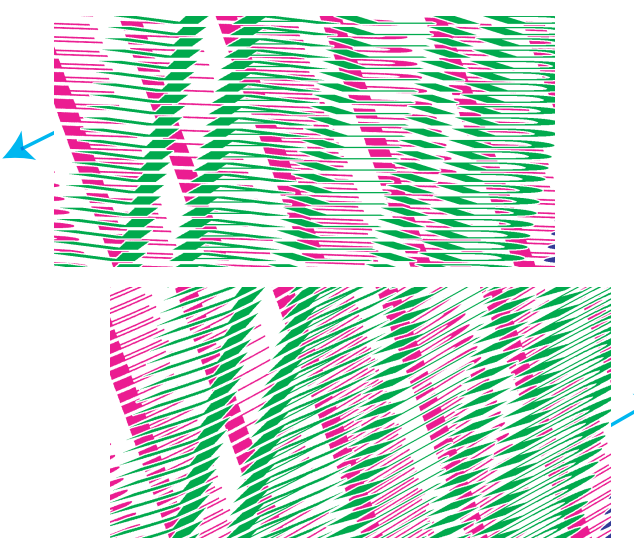

Fig. 14. Base and revealing layers transformed according to cosinusoidal functions, yielding a rectilinear moiré image. The moiré image remains rectilinear upon vertical translation of the revealing layer.
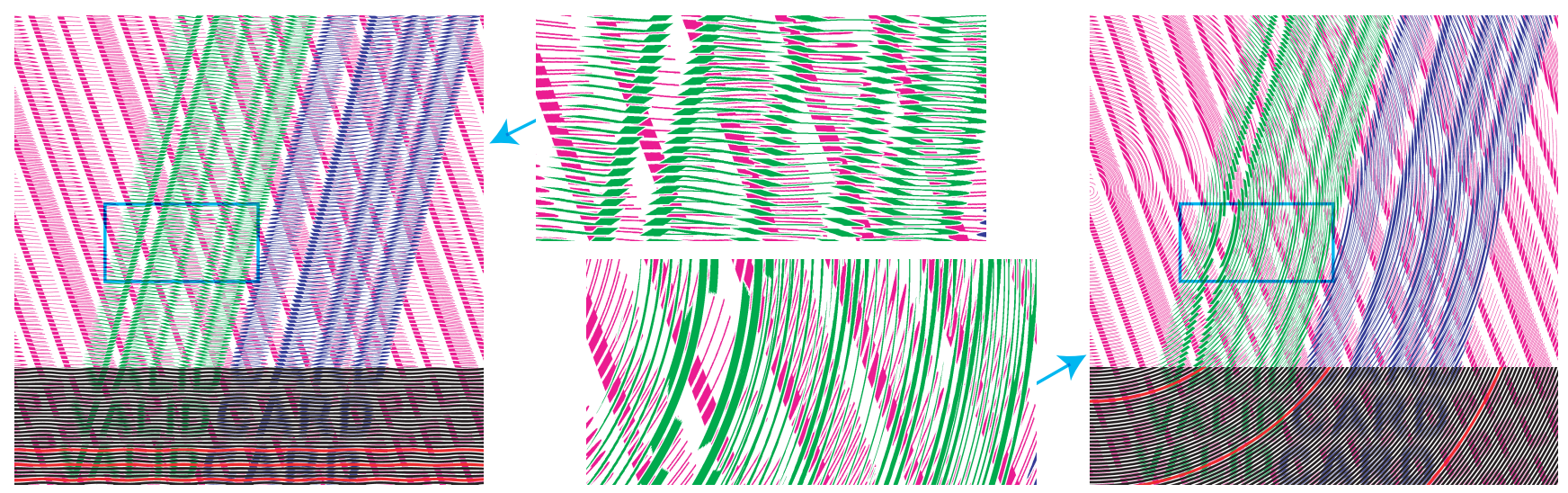

Fig. 15. Transformed base and revealing layers incorporating small Fig. 16. Strongly curved base band layer and perfectly circular periodic variations in $\mathrm{x}$ and $\mathrm{y}$. Upon translation of the revealing revealing layer yielding a rectilinear moiré image. Upon layer, the moiré image is subject to a periodic deformation. translation of the revealing layer, the moiré image deforms itself.

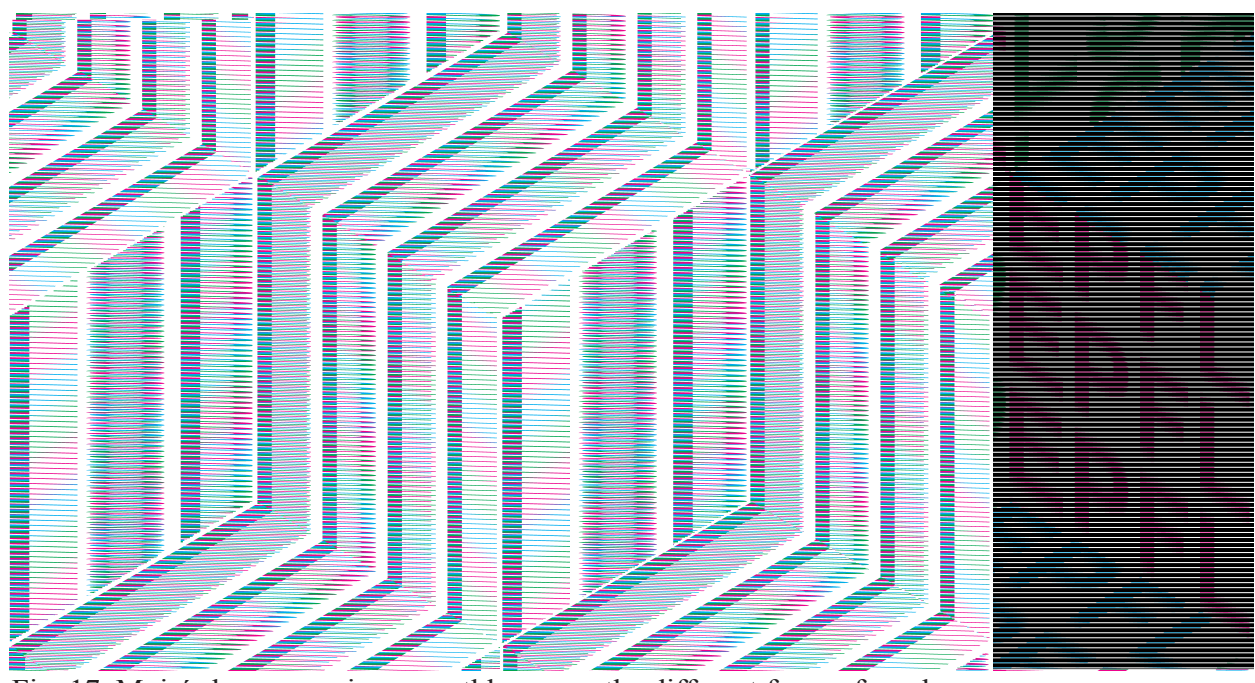

Fig. 17. Moiré shapes moving smoothly across the different faces of a cube upon translation of the revealing layer.
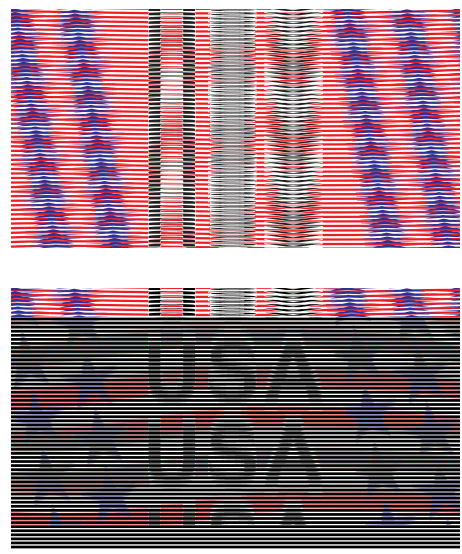

Fig. 18. Dynamic design inspired by the US flag with moiré image components moving according to different orientations and speeds.

The revealing layer file (.pdf) can be downloaded from http://diwww.epfl.ch/w31sp/publications/moire/bmi.html 
.A I .gi'

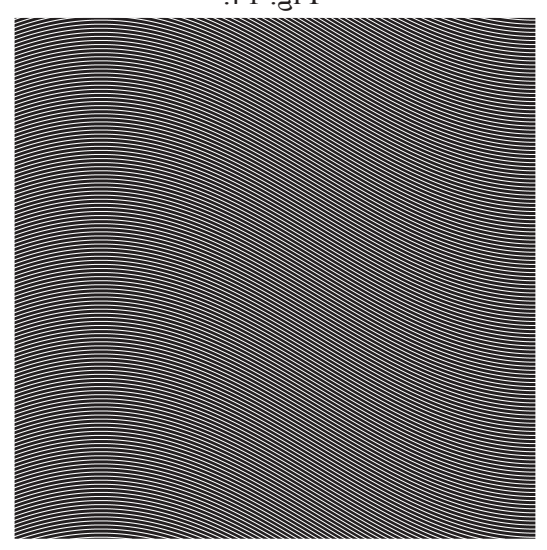

.дІ .gї

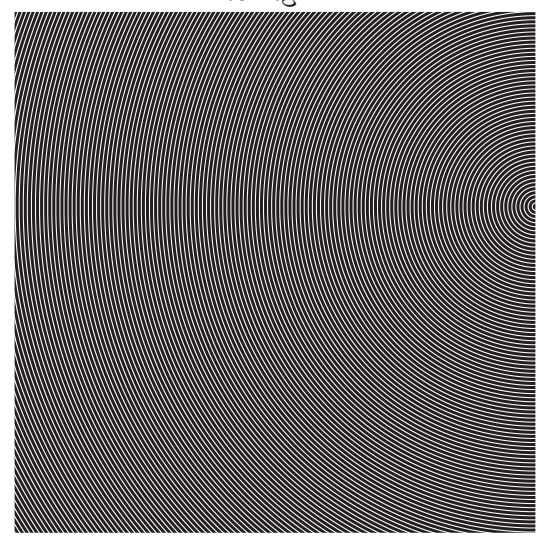

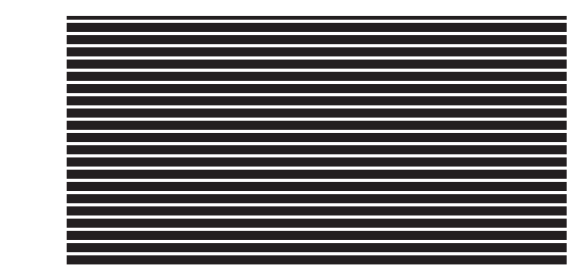

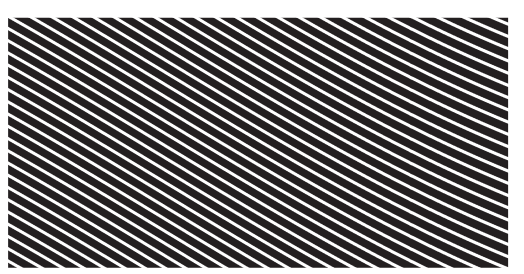

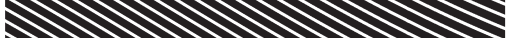

.हI .giन

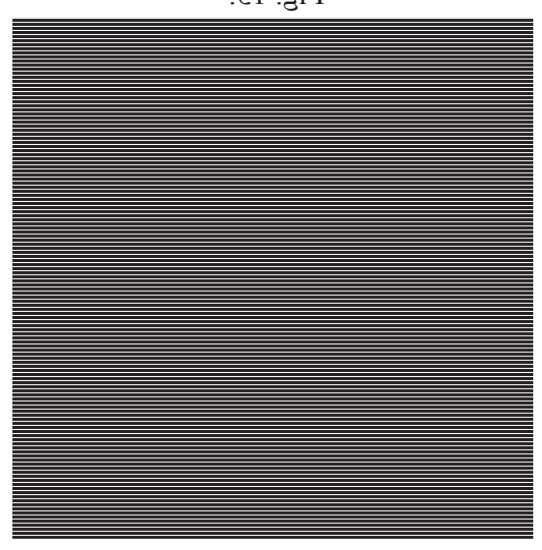

¿І .giन

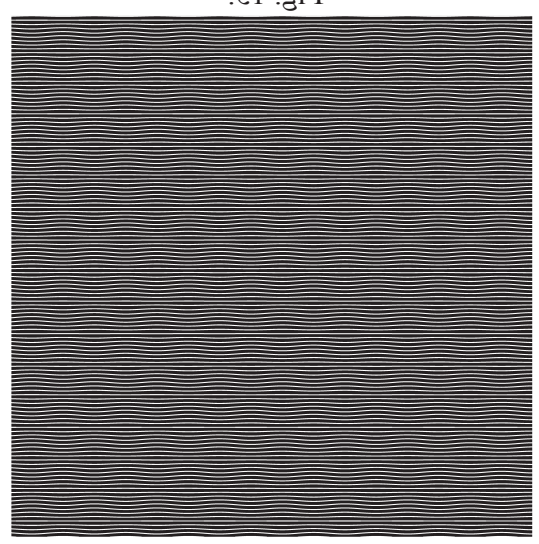

ГI .gi'
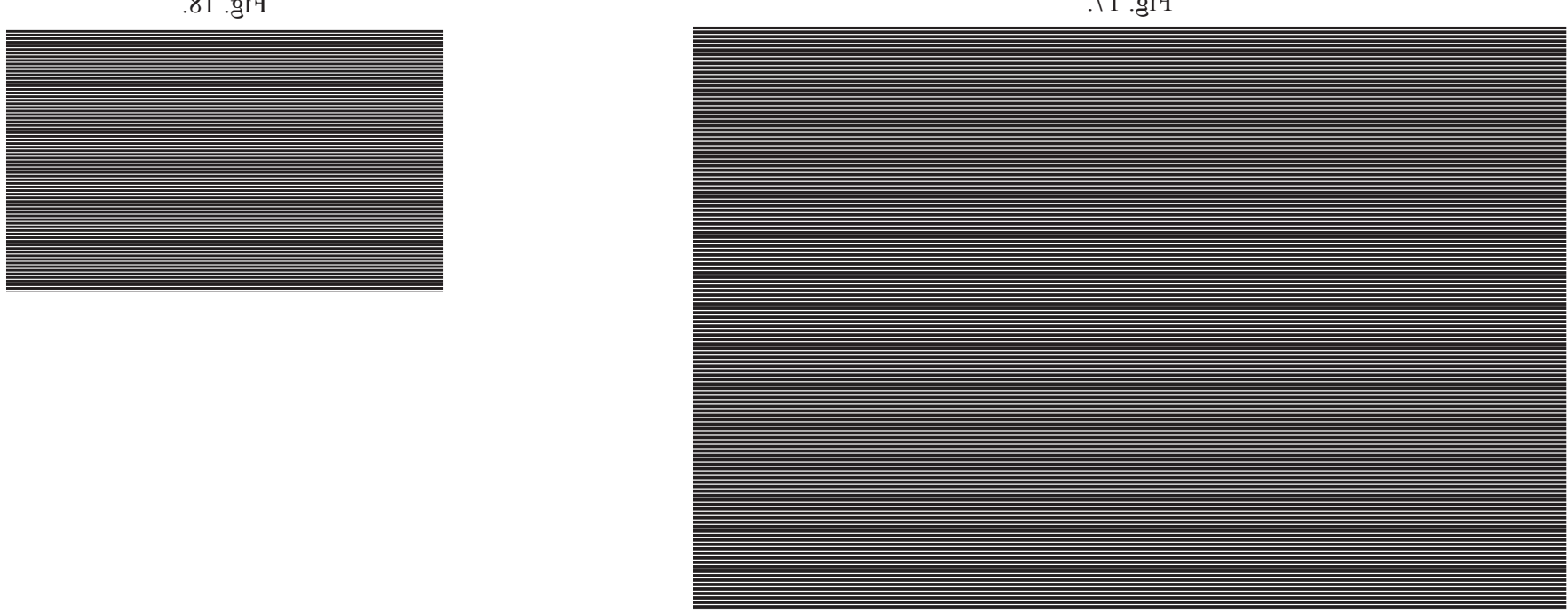

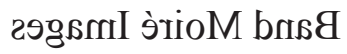

